\title{
Revisión Docencia y. en pandemia desde la carrera de Comunicación de la Universidad Estatal de Bolívar
}

Eduardo Albán malban@ueb.edu.ec

Margoth Chávez emchavez国ueb.edu.ec

Miguel Ocaña

jocana@ueb.edu.ec

Sandra Paredes

sparedes国ueb.edu.ec

Recibido: 18 de julio de 2021 | Aceptado: 18 de octubre de 2021

DOl: https://doi.org/10.18272/pd.v5i1.2385

Referencia de este artículo:

Chávez, E., Ocaña, J., Albán, M., Paredes, S. (2021). Docencia y comunicación en pandemia desde la carrera de comunicación de la Universidad Estatal de Bolívar. \#PerDebate, volumen 5 (pp. 192-215). Quito: USFQ Press.

Eduardo Albán comunicador social y periodista. Consultor independiente en relaciones públicas, marketing y comunicación política. Docente de la Carrera de Comunicación de la Universidad Estatal de Bolívar.
Margoth Chávez ingeniera comercial con mención en Negocios Internacionales, Universidad de las Américas. Ph.D., Universidad Central de Caracas. Docente de la Carrera de Comunicación de la Universidad Estatal de Bolívar.

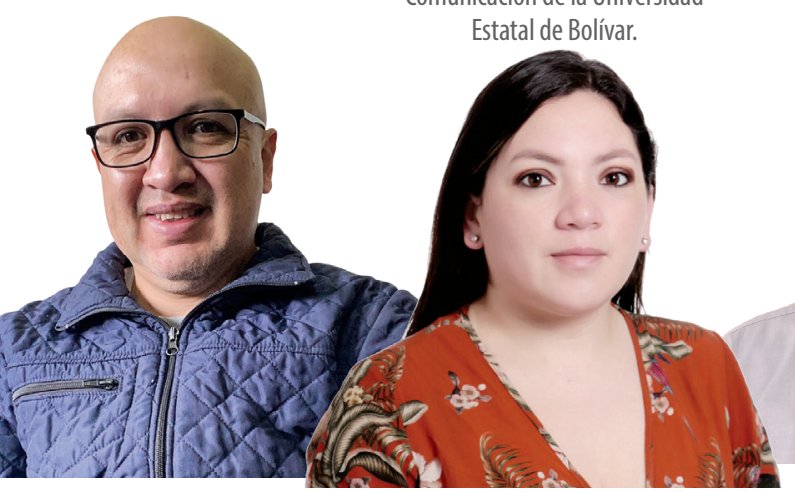

Miguel Ocaña periodista de medios de comunicación radial. Asesor y consultor de imagen. Docente de la Carrera de Comunicación de la Universidad Estatal de Bolívar.
Sandra Paredes periodista de medios impresos nacionales y corresponsal de noticias de medios internacionales. Docente de la Carrera de Comunicación de la Universidad Estatal de Bolívar.

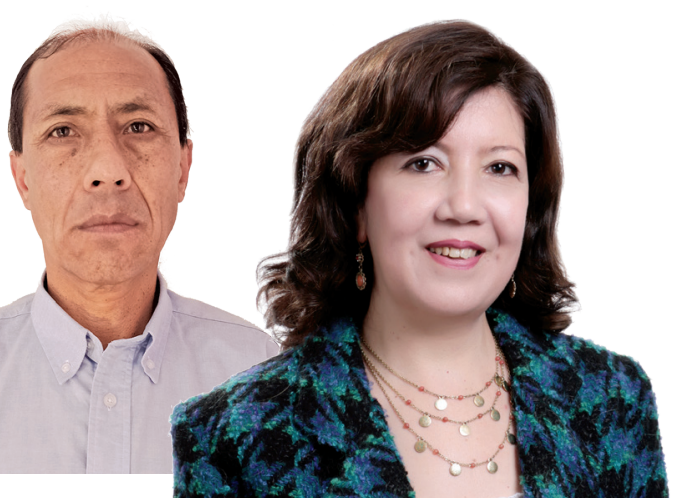




\begin{abstract}
Resumen
Esta investigación tuvo como objetivo analizar la actividad docente y su influencia en el nivel de comunicación en tiempos de pandemia en los estudiantes de la carrera de comunicación de la Universidad Estatal de Bolívar, desde tres ejes interdependientes: 1) Perfil psicográfico; 2) Relación clases virtuales docente-estudiante; 3) Factores psicosociales. Evidenció que los estudiantes que viven con más de tres personas han disminuido su nivel de concentración; la mayoría utiliza computadora y celular como medios de conectividad con internet fijo, complementando su educación a través de redes sociales. Se propone el uso de estrategias de educomunicación y un seguimiento psicológico a cada actor educativo. Como conclusión, la educación en tiempos de crisis sanitaria implica que no solo se deben crear condiciones tecnológicas apropiadas, sino el seguimiento psicológico por los entes pertinentes, para buscar la garantía de generar una cultura de resiliencia.
\end{abstract}

\title{
Palabras clave
}

conectividad, educación virtual, enseñanza-aprendizaje, IES, flujo de información

\section{Teaching and communication in pandemic from the communication career of the State University of Bolívar}

\begin{abstract}
This research aimed to analyze the teaching activity and its influence on the level of communication in times of pandemic in students of the communication career of the State University of Bolívar, from three interdependent axes: 1. Psychographic profile, 2. Class relationship virtual teacher / student and 3. Psychosocial factors. It was evidenced that students living with more than three people have decreased their level of concentration; Most use computers and cell phones as means of connectivity with fixed internet, complementing their education through social networks. The use of educommunication strategies and psychological monitoring of each educational actor are proposed. It was concluded that education in times of health crisis implies that not only appropriate technological conditions must be created, but also psychological monitoring by the relevant entities, to seek the guarantee of generating a culture of resilience.
\end{abstract}

\section{Keywords}

connectivity, virtual education, teaching-learning, IES, information flow 


\section{Introducción}

Esta investigación analiza la actividad docente y las principales relaciones de comunicación con los estudiantes en tiempos de la pandemia covid-19. Para el efecto, utiliza una encuesta virtual que abarca tres ejes de estudio: el perfil psicográfico, la relación entre el personal docente y estudiantil en clases virtuales, y, por último, los factores psicosociales en la etapa de confinamiento obligatorio. Esta encuesta se aplicó a los estudiantes de la Carrera de Comunicación de la Universidad Estatal de Bolívar, durante el período académico 2021.

Una de las tareas fundamentales es conocer la personalidad, el estilo de vida, intereses, aficiones de los estudiantes universitarios (explicados desde la perspectiva de varios autores). Asimismo, realiza una lectura previa de las experiencias vividas por el estudiantado en época de pandemia, con el fin de conocer las actividades desarrolladas en clases virtuales para medir si existe o no una buena relación entre docente y estudiante. Al final, identifica los factores psicosociales que enfrentan tanto docentes como estudiantes universitarios.

Estos hallazgos permiten comprender las dificultades que han vivido estos dos componentes de la educación universitaria en Ecuador, a raíz de la etapa de confinamiento obligatorio por causa de los contagios de covid-19.

\section{Un acercamiento conceptual al tema}

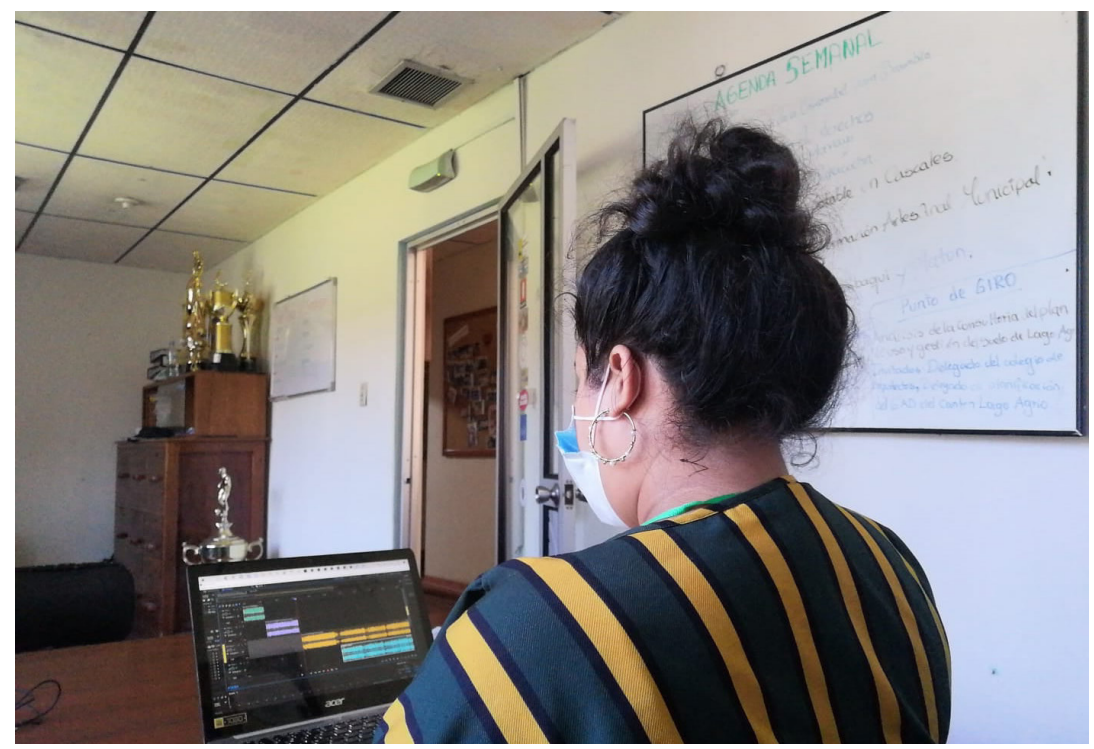

Fotografía 1. Los estudiantes tuvieron que acostumbrarse a estas nuevas formas de educación en tiempos de COVID-19. Fuente: Archivo Carrera Comunicación de la Universidad Estatal de Bolívar (UEB) 
A finales del 2019, el mundo se aprestaba a vivir una ola de contagios por causa de la covid-19, hecho que resultó universal y provocó que todas las naciones del mundo tomen medidas necesarias para evitar, no solo contagios, sino una gran cantidad de muertes. Según la Cepal (2020), el 11 de marzo de 2020, la OMS declaró que el planeta se encontraba en una etapa de pandemia tras el brote del virus SARS COV-2 causante de la enfermedad covid-19.

El titular de la OMS, Tedros Adhanom Ghebreyesus, comunicó al mundo sobre el elevado potencial de propagación internacional. La institución emitió las debidas alertas sobre el impacto y consecuencias en los sectores de salud, áreas sociales y también en la economía de las naciones. El virus SARS COV-2 tuvo su origen en China, en diciembre de 2019, desde ese mes ya se había propagado por más de 100 países en menos de dos semanas, y con una cifra que sobrepasaba los 100.000 casos, con más de 5.000 muertes.

En Ecuador, las principales autoridades gubernamentales tomaron acciones inmediatas para mitigar la llegada de la pandemia. Es así como la implementación de una serie de medidas relacionadas con la bioseguridad y control, consideradas como obligatorias, para proteger la salud de quienes habitamos en Ecuador. Se trata de las medidas de prevención y control mínimas que deben aplicarse durante la atención sanitaria de todos los pacientes para evitar la propagación de microorganismos patógenos que se transmiten a través de la sangre, los fluidos orgánicos o por medio de otras fuentes conocidas o desconocidas, según Informe del Ministerio de Salud Pública (2020).

El Ministerio de Salud Pública (MSP) viene trabajando, con el apoyo de la Organización Panamericana de la Salud y la Organización Mundial de la Salud (OPS/OMS), desde el mes de febrero de 2020, en las acciones de preparación para contener y responder a la pandemia. A partir del 29 de febrero de 2020, fecha de confirmación del primer caso positivo de covid-19 en Ecuador, se redoblaron los esfuerzos para el tratamiento de los casos, la vigilancia epidemiológica, el fortalecimiento de la capacidad de diagnóstico y laboratorios, así como la protección del personal de salud y la comunicación de riesgo dirigida a la comunidad (Naciones Unidas, 2020, p. 5).

La paralización de actividades fue inmediata. Solo el personal de trabajadores de la salud, de seguridad, medios de comunicación y funcionarios de gobierno de áreas sensibles tuvieron la posibilidad de movilizarse. El resto de la población debió guardar cuarentena obligatoria.

En torno a la situación económica que se vive en la región, y según informe del 2020, de la Comisión Económica para América Latina y el Caribe (Cepal), la situación social y económica que se vivía en la región ya se estaba 
deteriorando, ya existía un descontento social debido a las desigualdades particularmente en la salud, la educación y el desempleo (Cepal, 2020a, p. 1).

De acuerdo con el reporte macroeconómico del BID, en el texto "Políticas para combatir la pandemia", se prevé una caída en el valor de los bienes y servicios producidos por los países con impacto en los procesos básicos de construcción de capital humano, por lo que es importante que se incrementen políticas de Estado que eviten que los países tengan problemas de liquidez y no tengan que pasar por una deflación en el nivel de los precios de bienes y servicios (BID 2020a, p. 5).

Acerca del campo educativo, los acuerdos ministeriales de educación y salud pública tomaron como referencia lo que señala la Constitución de Ecuador (2008, art. 26) sobre el derecho que tienen todas las personas y el deber ineludible e inexcusable del Estado para apoyar a este sector.

El artículo 3, numeral 1 de la Constitución, prevé como deber primordial del Estado: "Garantizar sin discriminación alguna el efectivo goce de los derechos establecidos en la Constitución y en los instrumentos internacionales, en particular la educación [...]" (Constitución, 2008).

La normativa del Consejo de Educación Superior (CES) establece que los recursos de aprendizaje en tecnologías digitales deberían estar disponibles, tanto para el personal académico como para los estudiantes. En el caso de que estos recursos no estuvieran disponibles, las instituciones de educación superior (IES) debían posibilitar a los estudiantes la oportunidad de tomar el curso en otro momento.

La Unesco ha identificado diferentes brechas en la educación; entre ellas, la desigualdad en la asignación de personal académico calificado para atender a las poblaciones rurales, considerando que en estas zonas no existen suficientes recursos económicos para contratar docentes que impartan conocimientos en las poblaciones indígenas y migrantes. Son muchas las medidas de protección ante la pandemia que los países han adoptado, entre ellas, la suspensión de las clases presenciales en todos los niveles educativos (escuelas, colegios, universidades) y la utilización de formatos y plataformas, la atención médica y el bienestar integral de docentes y estudiantes (Unesco, 2016; Messina y García, 2020, p. 1).

\section{El perfil psicográfico del estudiante}

El concepto psicográfico hace referencia a la personalidad, estilo de vida, intereses, aficiones y valores. Para entender este término se hace referencia a la Real Academia Española (2010), la cual define personalidad como la "Diferencia 
individual que constituye a cada persona y la distingue de otra", pero también como el "Conjunto de características o cualidades individuales que destacan en algunas personas".

Etimológicamente, el término personalidad se deriva de la expresión latina persona, que precede a su vez del griego cuyo significado es mirada, aspecto exterior, apariencia, imagen percibida por los demás, máscara, vestimenta alrededor del cuerpo y todo aquello que sirve para arreglarse con el fin de mostrarse en público y que encuentra en el mundo del teatro su aplicación principal.

Asimismo, la personalidad hace referencia a un conjunto de rasgos o características, particularidades que definen a las personas. Según el estudio titulado Perfiles psicológicos y académicos de estudiantes universitarios, realizado en la Universidad de Mar del Plata, donde se evalúo la personalidad, los procesos cognitivos básicos (que están relacionados con la memoria) el nivel de bienestar psicológico y las características demográficas (sexo, edad, nivel educativo de los padres y tipo de carrera que se cursa) se pudo determinar que las universidades deben trabajar desde la atención primaria de la salud, precautelando la salud mental de los estudiantes, además se deben implementar políticas públicas para identificar la problemática y los factores de riesgo por las que atraviesan los estudiantes universitarios. También se manifiesta en esta investigación que se deben establecer programas para la Salud y la importancia de mantener datos estadísticos sobre los casos epidemiológicos en la población estudiantil (Cibanal, 2014).

En la investigación titulada Salud mental percibida y distrés psicológico en estudiantes de Ciencias Psicológicas y Médicas de la Universidad Central del Ecuador se aplicó una encuesta a 300 estudiantes universitarios. Según los resultados obtenidos, de forma independiente tanto el nivel de salud mental, medido a través de sus cuatro dimensiones, así como el nivel de distrés psicológico, medido a través de sus tres dimensiones generales, han sido mayoritariamente percibidos en el nivel medio o moderado, obteniéndose una correlación positiva moderada entre estas variables $(r=, 490 ; p=0,05)$. En conclusión, mientras la percepción de salud mental es más alta, menor es la presencia de síntomas de distrés psicológico (García, et al., 2019).

En torno al estilo de vida, intereses, aficiones y valores de los estudiantes universitarios, Rodríguez (2015) manifiesta que entre las características de los estudiantes de hoy se encuentran las Ilamadas generaciones. Los aprendices forman parte de la generación Y, conocida como nativos digitales, cuyas características peculiares los identifican como seres solitarios, inmersos en una situación de crisis económica y dependientes, pragmáticos en sus estudios y con una gran capacidad para obtener información. 


\section{Relación clases virtuales docente / estudiante}

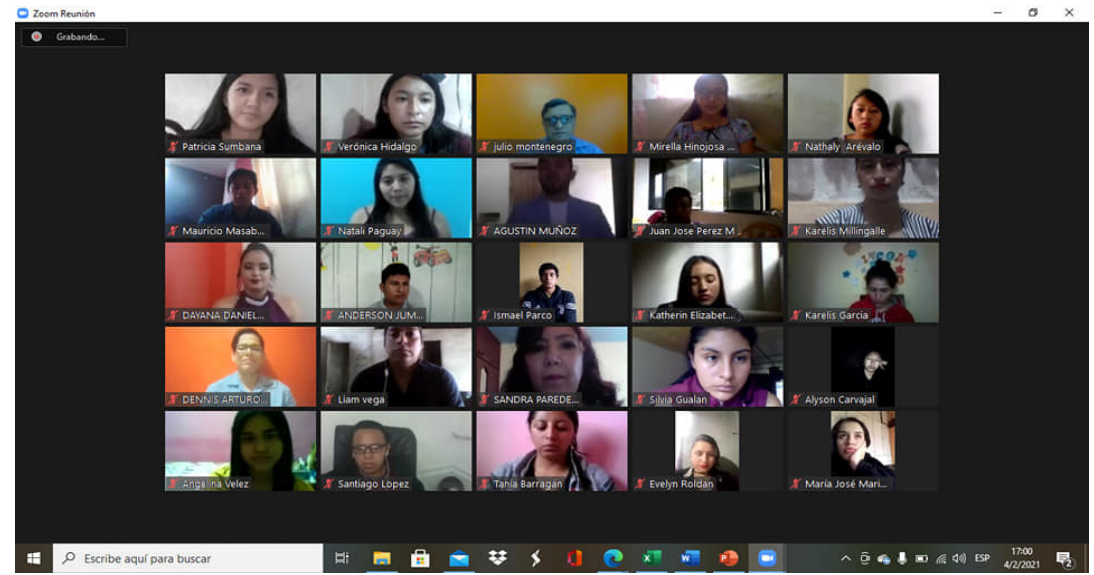

Fotografía 2. Los estudiantes se adaptaron a las actividades académicas virtuales. Fuente: Cuenta de Facebook de la Carrera de Comunicación UEB

En medio de los cambios tecnológicos que se viven en el mundo actual, (Pedraja et al., 2006) sostienen que la sociedad del conocimiento se está construyendo con base en las modernas tecnologías de la información y de la comunicación (TIC). Esta se caracteriza por una estructura económica y social en la que el conocimiento sustituye al trabajo, a las materias primas y al capital como una fuente más importante en la productividad, el crecimiento y las desigualdades sociales.

Mientras más se incrementa la accesibilidad de las personas a la información se hace necesario que se tome al estudiante como coprotagonista de su aprendizaje, donde la tecnología se convierta en un instrumento, en un recurso técnico o procedimiento empleado para estudiar en la virtualidad (Rodríguez, s.f., p. 4).

La enseñanza ha evolucionado a lo largo de la historia, iniciando con la educación por correspondencia la cual consiste en la utilización de textos de conocimientos básicos que le permitían al docente interactuar con el estudiante durante las clases, hasta llegar a la enseñanza telemática que se promueve a través del uso del ordenador donde se integran programas educativos con el fin de adquirir nuevas y distintas adaptaciones de flexibilidad en beneficio de docentes y estudiantes (García et al., 2019).

La educación virtual, conocida también como educación en línea, desarrolla una relación de enseñanza y aprendizaje de forma on-line, mediante la cual el estudiante y el docente pueden interactuar sin la presencia directa 
del tutor, modalidad que permite que estudiantes y docentes puedan gestionar su tiempo. En este sentido, compara al término virtualidad con la fuerza o la potencia; se trata de un término usado en informática para hablar de realidad construida por medio de sistemas informáticos o digitales; se lo podría comparar con la realidad virtual que permite transportarse a través del ciberespacio de un entorno a otro en cuestión de segundos. La sociedad está exigiendo un cambio en el enfoque de la educación por intermedio del uso de herramientas virtuales como es la web 2.0, instrumento que aporta en el desarrollo del conocimiento, a través de la búsqueda, recopilación, gestión y reflexión y prácticas de aprendizaje entre el docente y el estudiante (Mota et al., 2020).

La utilización de las TIC aplicadas a los contextos de formación mejoran los procesos de aprendizaje entre el estudiantado porque permite su participación directa, además de la integración entre grupos de trabajo, de la habilidad de liderazgo y participación entre sus pares. Esto permite que los estudiantes sean capaces de asumir nuevos retos profesionales. La virtualización se define como el movimiento inverso a la actualización, por cuanto lo concibe como el paso de lo actual a lo virtual. En este sentido se puede manifestar que la virtualidad siempre ha estado en nuestras vidas, solo que actualmente la utilizamos como instrumento en la mayoría de nuestras tareas cotidianas hasta las actividades académicas.

El conocimiento es personalizado y está disponible en red. Eso posibilita un nuevo paradigma de enseñanza-aprendizaje existiendo una mezcla entre teorías y metodologías provenientes de la pedagogía, psicología y sociología; todo esto con el propósito de mejorar las condiciones para el aprendizaje de los estudiantes (Martínez y Torres, 2020).

La transición de la presencialidad a la virtualidad ha demandado que estas deban acceder a diferentes herramientas virtuales entre ellas la web 2.0, además han tenido que acceder a los denominados Entornos Virtuales Abiertos a través de los cuales los estudiantes tienen la oportunidad de recibir conocimientos de parte de importantes ponentes, a los cuales eran difíciles de contactar durante las clases presenciales, y en la actualidad gracias a los webinars (contenido formativo en formato vídeo que se imparte a través de internet). (Aguilar et al., 2016)

También está el rol del docente (Ryan et al., 2000 citado en Martínez y Ávila 2014) que se transforma en el mediador o intermediario entre los contenidos y la actividad constructivista que despliegan los alumnos para asimilarlos. Los roles y responsabilidades del docente en línea se pueden agrupar en cuatro categorías: pedagógica, social, administrativa y técnica. 


\section{Los factores psicosociales}

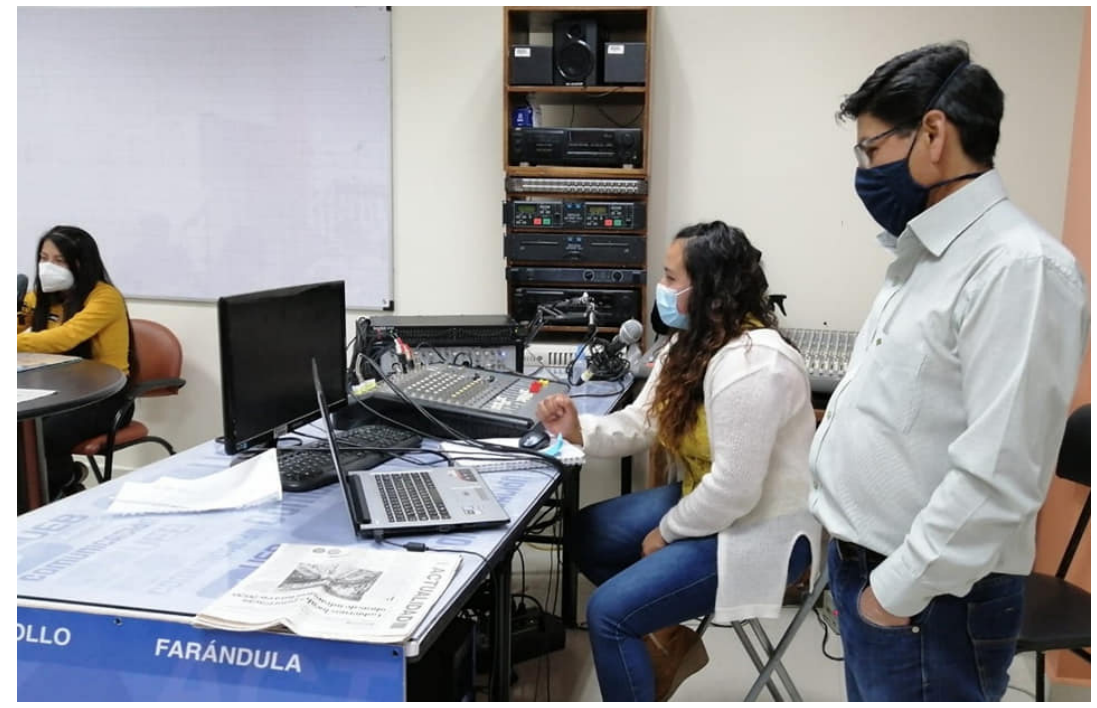

Fotografía 3. Las medidas de mitigación para prevenir la covid-19 se redujeron y los estudiantes pudieron asistir de forma presencial. Fuente: Cuenta Facebook Carrera de Comunicación UEB

En los últimos años, con las nuevas exigencias académicas, laborales, sociales y profesionales, los estudiantes universitarios se han enfrentado a una serie de retos que les demandan mayor cantidad de tiempo, esfuerzo y dedicación en esta época competitiva (Armenta, 2019). Quienes inician sus estudios universitarios, deben saber que implica una responsabilidad de alto peso, pues, aunado al rendimiento académico, están las metas y objetivos que se establezcan, además del cumplimiento responsable en cada una de sus asignaturas.

El estrés se ha manejado históricamente para denominar realidades muy distintas. Es un concepto complicado, vigente y atrayente. Por tal razón, Martínez y Díaz citado en Armenta et al., 2020, p. 403) afirman que el fenómeno del estrés se lo suele interpretar en relación con una amplia gama de experiencias; entre ellas se destaca nerviosismo, tensión, cansancio, agobio, inquietud y otras sensaciones similares, como la presión escolar, laboral o de otra condición. De igual manera, se lo atribuye a situaciones de miedo, temor, angustia, pánico, afán por cumplir, vacío existencial, celeridad por cumplir metas y propósitos, incapacidad de afrontamiento o incompetencia interrelacional en la socialización (Valero et al., 2020, p. 64).

También puede ser definido como la acumulación de situaciones sociales conflictivas o las presiones del ambiente que excede la capacidad de las personas 
para adaptarse a las mismas (Estramiana et al., 2010 citado Armenta et al., 2020, p. 403). La palabra estrés es utilizada durante ya varios años para denominar experiencias diferentes, porque en ellas se desarrollan nerviosismo, tensión, agobio, cansancio, inquietud, entre otras emociones que forman parte del estrés (Armenta y Quiroz, 2019).

En ese sentido, Reyes y Trujillo (2020) manifiesta la situación de estrés, ansiedad, depresión e ira que viven los estudiantes ecuatorianos, a partir del 14 de marzo del 2020. Viven en un estado de alarma constante como consecuencia de la pandemia que azota a nuestro país y al mundo entero; además, está el relato etnográfico en el que los estudiantes deben vivir por la cuarentena, al tener que compartir espacios reducidos que inciden en su rendimiento académico.

\section{Materiales y métodos}

El tipo de investigación utilizado fue descriptivo con un enfoque cuantitativo y un método analítico. Como técnica de recolección de datos primarios se aplicó una encuesta digital y, posteriormente, se llevó a cabo un análisis e interpretación de datos.

La investigación descriptiva trata de realizar una narración de la manera más clara posible de algún suceso o evento dado, para lo cual en este caso sirvió para identificar las percepciones de los estudiantes. Así, se aplicó la descripción de cada una de las variables de estudio, conjuntamente con los hallazgos obtenidos.

Con la utilización del método analítico se analizó el impacto que tiene la comunicación y la docencia en la carrera de Comunicación de la Universidad Estatal de Bolívar, para indagar las causas, sus efectos y, finalmente, presentar propuestas para aportar soluciones al problema.

Como técnica de recopilación de datos, la aplicación de una encuesta digital permitió recoger información primaria y determinar la percepción de los estudiantes de la carrera de Comunicación sobre nivel y profundización de la dinámica comunicativa en tiempos de pandemia. La población correspondió al total de los individuos o elementos a quienes se refiere la investigación; es decir, todos los elementos estudiados, por ello también se le llama universo. De acuerdo con Arias (2012), la población es el conjunto de personas que intervienen en la investigación; en este caso, los estudiantes de la carrera indicada.

Se efectuó un muestreo por conveniencia de los investigadores que, según Malhotra (2008), es una técnica de muestreo no probabilístico que busca obtener una muestra de elementos convenientes, adicional por la facilidad de manipulación de datos y por el número finito de la población (439 estudiantes). 
Por eso, se realizó la convocatoria para llenar la encuesta digital a todos los estudiantes de la carrera, obteniendo 228 respuestas.

Durante la etapa de recolección de datos se utilizó Google forms que es un software de administración de encuestas en la web que ofrece Google. Seguidamente, se recurrió a Microsoft Excel para el registro y tabulación de datos. Finalmente, en el procesador de datos Word se analizaron e interpretaron los resultados mediante un método analítico, para exponer de manera explícita el estudio de los datos recopilados.

\section{Resultados}

Los 228 estudiantes participantes fueron consultados en función de tres componentes: el primer bloque analiza el perfil psicográfico; el segundo bloque indaga la relación que se da entre el estudiante y el docente, y el tercer bloque busca identificar los factores psicosociales de los estudiantes, a raíz de los síntomas causados por el aislamiento obligatorio o por haberse contagiado de covid-19.

De los encuestados, 228 en total, 142 son mujeres y 86 son hombres (ver Figura 1).

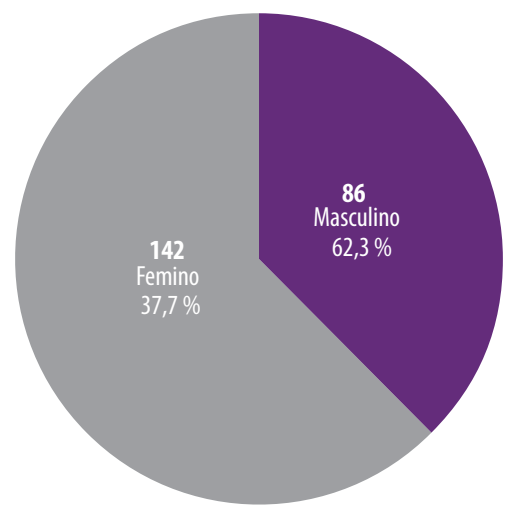

Figura 1. Género. Fuente y elaboración propias

Las edades de los encuestados oscilan entre 18-21 años (47,4 \%), 22-25 años (43,4 \%), 26-29 (7 \%) y el 2,2 \% para mayores de 30 años. No existen estudiantes menores a 18 años (ver Figura 2). 


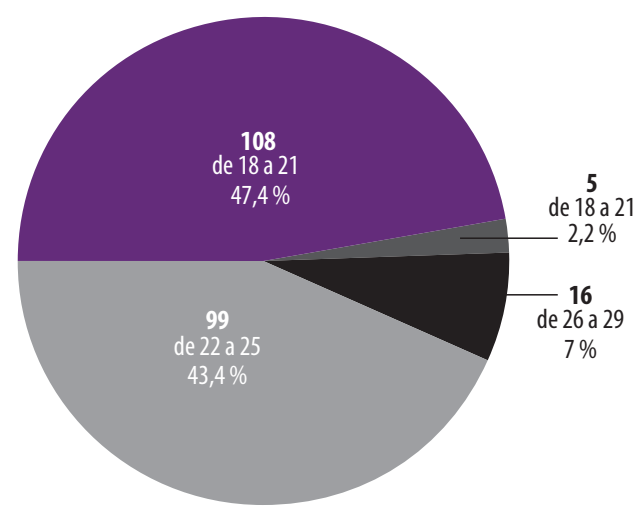

Figura 2. Edad. Fuente y elaboración propias

De los 228 estudiantes, el 73,6\% se dedica exclusivamente a las actividades académicas, mientras que el $19,8 \%$ cuenta con un trabajo remunerado como una actividad laboral (ver Figura 3).

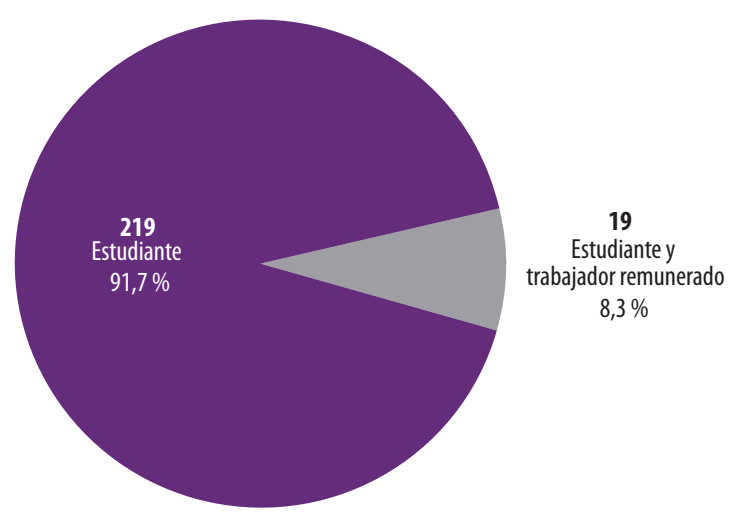

Figura 3. Ocupación. Fuente y elaboración propias

En cuanto al lugar de residencia referido por los participantes durante el confinamiento por la pandemia de la covid-19, este se distribuyó así: 73,6 \% en la región Sierra, 19,8 \% en la región Costa, 6,2 \% en la Amazonía y 0,4 \% en la región Insular (ver Figura 4). 


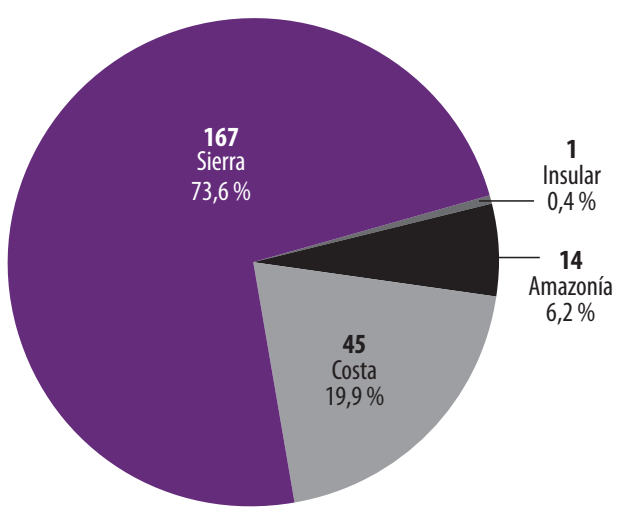

Figura 4. Lugar de vivienda. Fuente y elaboración propias

Con respecto al número de personas que conviven en el mismo hogar con los estudiantes durante el confinamiento, se observó que el $69,7 \%$ vive con más de tres personas, $13,2 \%$ vive con tres personas, $8,8 \%$ vive con dos personas, $5,3 \%$ con una persona y $3,1 \%$ vive solo (ver Figura 5 ).

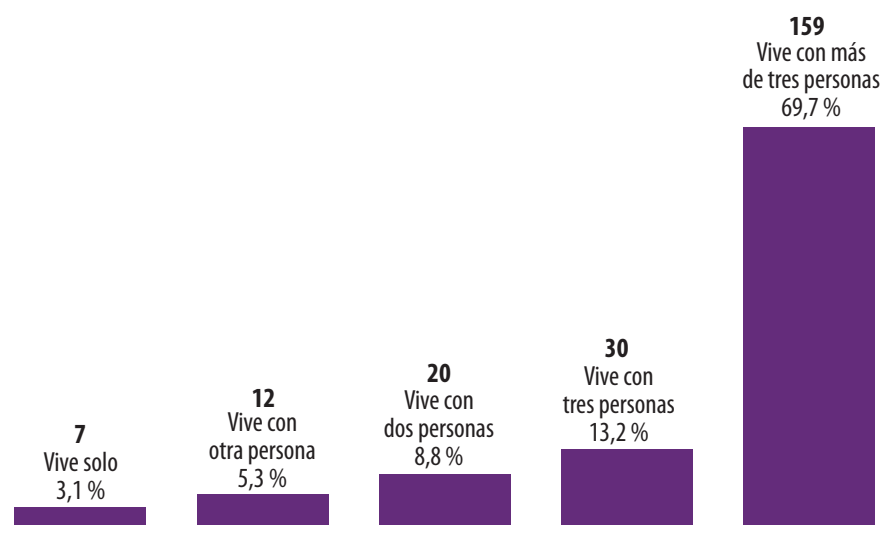

Figura 5. Vivienda. Fuente y elaboración propias

Los datos muestran que el acceso a las clases virtuales se da de esta manera: $47,6 \%$ por medio de una computadora personal, $43,6 \%$ a través de un teléfono móvil; mientras que el $8,4 \%$ ingresa desde un computador de escritorio y $0,4 \%$ desde una tableta (ver Figura 6). 


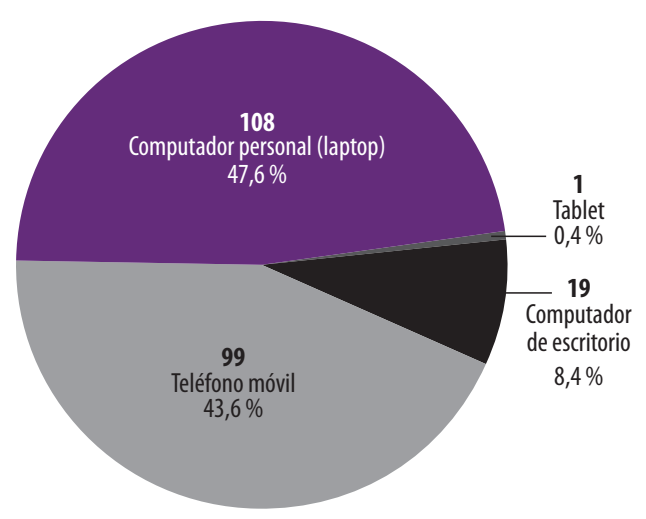

Figura 6. Acceso a clases. Fuente y elaboración propias

En cuanto al tipo de conexión, el 73,6 \% (167 estudiantes) utiliza internet desde un punto de conexión fija; en cambio, el $26,4 \%$ (60 estudiantes) acceden mediante un paquete de datos móviles (ver Figura 7).

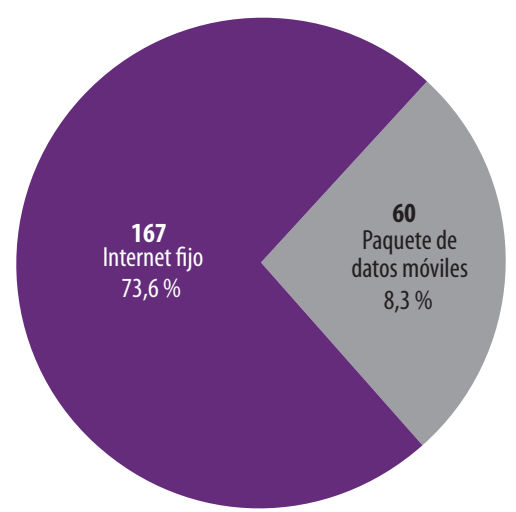

Figura 7. Tipos de conexión. Fuente y elaboración propias

Acerca de los canales de comunicación del docente, el 70,9\% de los estudiantes utiliza grupos de WhatsApp, el 15,4\% la plataforma EVEA. El 11,4\% lo hace por medios oficiales (correo electrónico institucional) y el $2 \%$ a través de redes sociales (Facebook o Instagram) (ver Figura 8). 
161

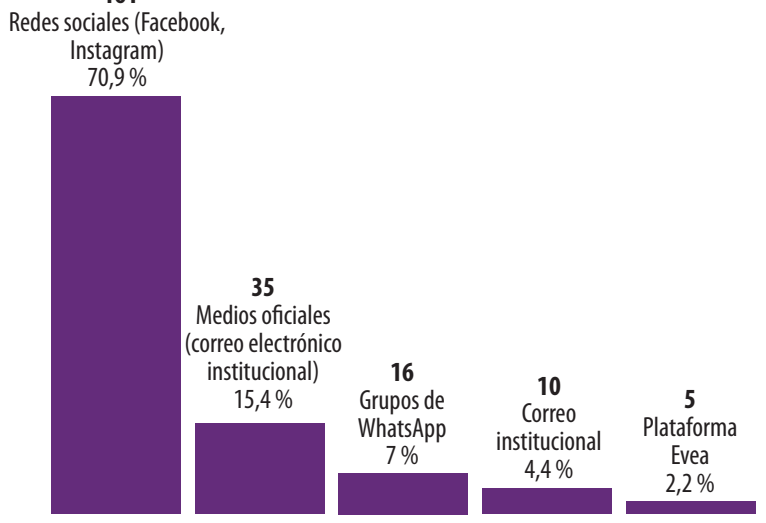

Figura 8. Comunicación con el docente. Fuente y elaboración propias

Respecto a la valoración del flujo de información, el 52,9 \% lo considera medianamente bueno ("más o menos"), el 42,3\% responde "bueno" y para el 4,8\% es "malo" (ver Figura 9).

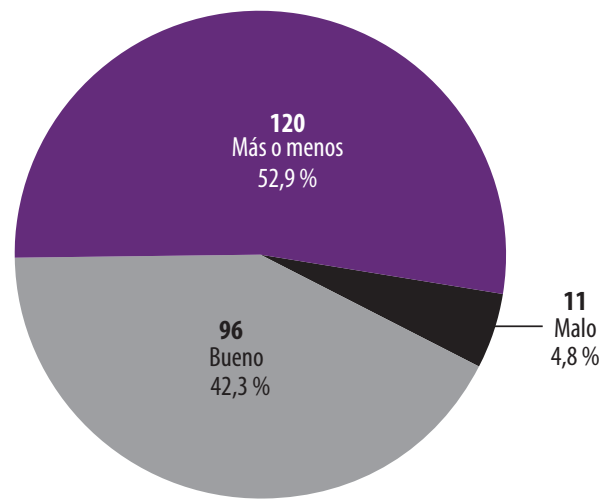

Figura 9. Formas de comunicación con el docente. Fuente y elaboración propias

La Figura 10 presenta resultados sobre la incorporación de nuevas herramientas para comunicarse entre el personal docente y estudiantes, a diferencia de la educación presencial. El 89,5\% responde "Sí" y el 10,5 \% "No". 


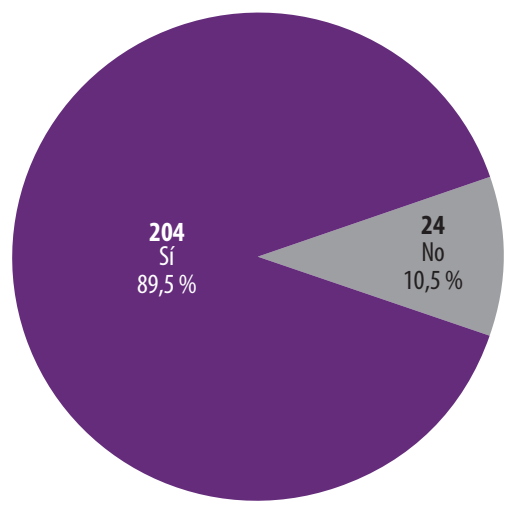

Figura 10. El docente utiliza medios para comunicarse con los estudiantes. Fuente y elaboración propias

El tercer bloque de preguntas indaga la situación psicológica y social del estudiantado en medio de la pandemia por causa de la covid-19 o por el aislamiento obligatorio. El 26,2 \% de los estudiantes sufre de dolores de cabeza, el $18,6 \%$ tiene crisis de ansiedad e insomnio, el $17,2 \%$ siente agotamiento y se encuentra sin fuerzas. El 13,6\% se encuentra preocupado, el 7,2 \% tiene la sensación de estar enfermo. El $9 \%$ no se siente saludable y el 8,1 \% no puede conciliar el sueño (ver Figura 11).

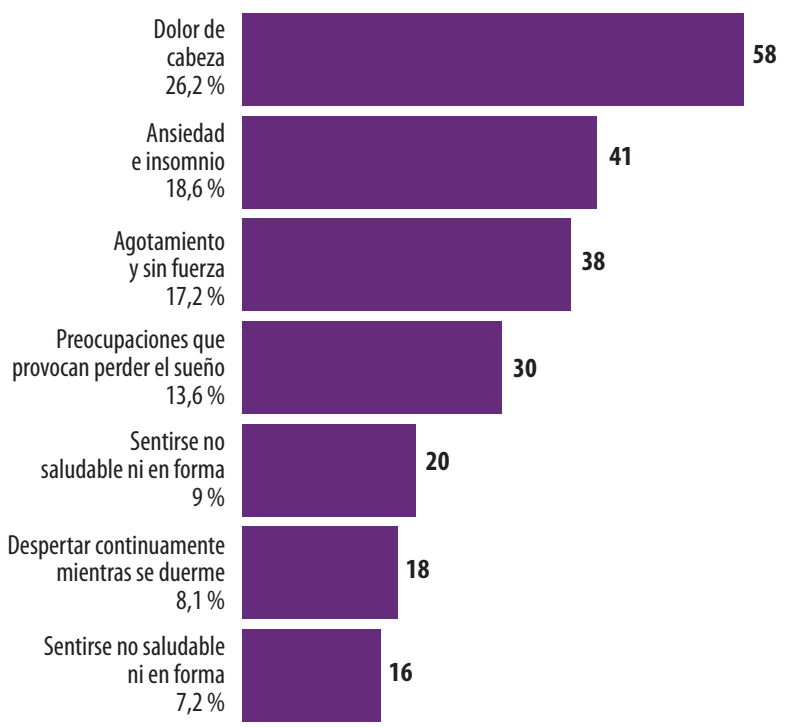

Figura 11. Síntomas psicosomáticos en pandemia. Fuente y elaboración propias 
En la cotidianidad al 52,3\% le cuesta mucho más tiempo realizar las actividades; el $25,7 \%$ se siente aburrido y con poca satisfacción sobre las actividades que realiza. El 16,7 \% siente mucha satisfacción y alegría al momento de cumplir con sus actividades. Finalmente, el 5,4 \% se siente menos ocupado y activo (ver Figura 12).

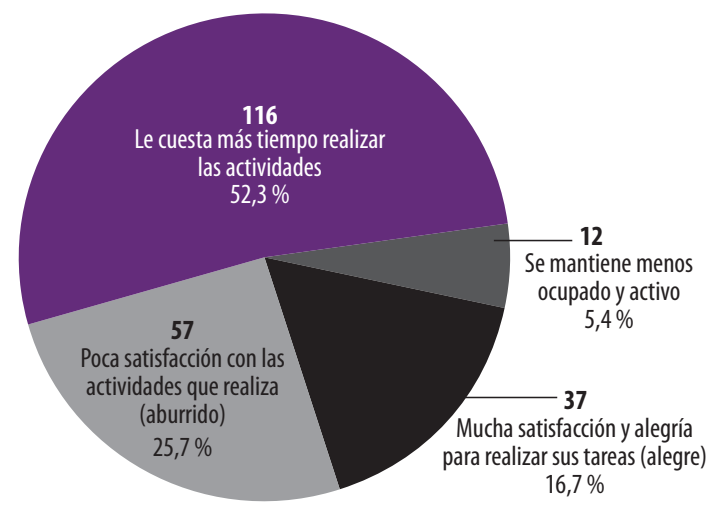

Figura 12. Situación diaria. Fuente y elaboración propias

La última pregunta está relacionada con la depresión, para indagar la opinión sobre este problema. El $27,4 \%$ señala que es una percepción de bajo valor personal; el $24,2 \%$ considera que es la incapacidad de estar activo por un alto grado de nerviosidad. El 21,1\% responde que es el sentimiento que no vale la pena vivir, y el 14,8\% dice que son deseos de morir (ver Figura 13).

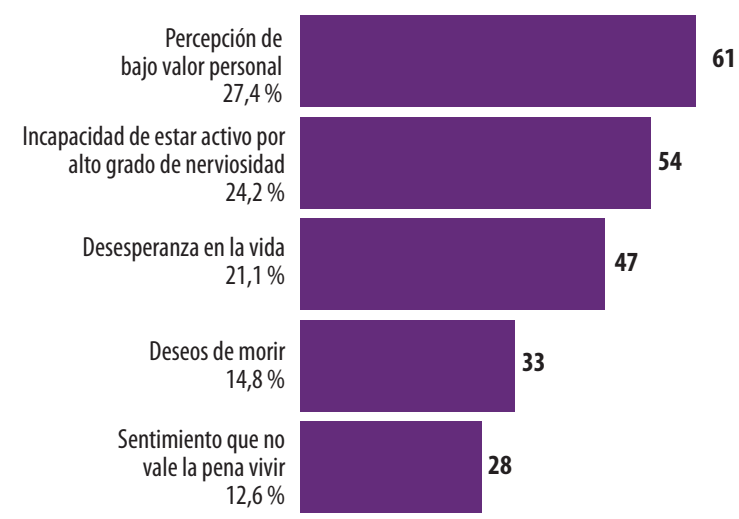

Figura 13. Opinión acerca de la depresión. Fuente y elaboración propias 


\section{Discusión}

La encuesta planteada buscaba recolectar tres tipos de datos: la relación que se da entre el docente y los estudiantes en tiempos de pandemia, la actividad académica de los estudiantes y las actitudes que asumen los alumnos frente a la educación virtual, teniendo como punto de comparación la educación presencial. Un primer componente de los resultados muestra la situación geográfica, las características de la vivienda en la que residen, edad, género, ocupación. Cabe destacar, de entre los resultados, que la mayoría de estudiantes viven en la región Sierra y sus edades oscilan entre los 21 y 25 años; la mayoría se dedica exclusivamente a las actividades académicas.

Un segundo componente de la encuesta señala las formas en que el estudiantado accede a sus clases. Se destaca y llama la atención que la mayoría lo hace por medio de su computador personal y desde su teléfono móvil, lo cual (en el segundo caso) derivaría en problemas de atención y concentración durante las actividades académicas. A estos datos se suma que la mayoría de estudiantes cuenta con una conexión de internet fija y un pequeño porcentaje lo hace a través del pago de servicios de internet móvil o en el celular.

El estudiantado de la Universidad Estatal de Bolívar se comunica permanentemente con el personal docente mediante WhatsApp, una aplicación de mensajería instantánea de uso popular y de fácil acceso para las personas. A pesar de la campaña de uso de la plataforma EVEA por parte de las autoridades de la universidad, un porcentaje mínimo de estudiantes la utiliza, por lo que estos resultados podrían ser de utilidad a futuro para emprender campañas de comunicación y socialización para el uso de la plataforma oficial de esta institución de educación superior.

A diferencia de la educación presencial, el estudiantado asegura que existe una buena predisposición del personal docente por mejorar los canales de comunicación. Sin embargo, para un buen porcentaje de estudiantes, la relación con sus docentes es medianamente buena.

Finalmente, los resultados muestran que el ambiente de los estudiantes en tiempos de pandemia no fue el adecuado, sobre todo por las limitaciones generadas desde el Gobierno para evitar contagios por la covid-19. La encuesta da a conocer una serie de factores psicosomáticos claramente identificados por el estudiantado, quienes reconocen que sufren de dolores de cabeza recurrentes, crisis de ansiedad relacionadas con insomnio, preocupación, falta de sueño; además, sienten agotamiento durante el desarrollo de sus actividades. Si bien algunos pueden conciliar el sueño, es muy común que se despierten continuamente mientras duermen en las noches, producto del agotamiento o de esa sensación de estar enfermos. 
Durante el desarrollo de sus actividades, una buena mayoría de estudiantes señala que le cuesta mucho más tiempo realizar sus labores diarias, en comparación con años anteriores en tiempos de normalidad. Un porcentaje considerable se siente aburrido de la cotidianidad, así como poco satisfecho de lo que realiza.

La encuesta buscó interpretaciones del término depresión y un buen número de participantes coincide en definirla como un sentimiento de desesperanza o falta de ganas de vivir, como una actitud de bajo valor personal, esa incapacidad para estar activo permanentemente y superar las etapas de nerviosismo que son comunes en estados de depresión aguda.

\section{Conclusiones}

La covid-19, una enfermedad de alto contagio que se convirtió en pandemia mundial, llegó a cambiar la cotidianidad de los seres humanos. El aislamiento obligatorio para evitar su propagación resignificó la forma en que se relacionan las personas. Atrás quedaron las formas de contacto más habituales utilizadas desde siempre, porque se activaron el teletrabajo, la telemedicina, las compras por internet y la teleeducación. Por esa razón, se concluye que quien no logró adaptarse a estos cambios, no pudo superar esta etapa de pandemia.

Las universidades y otros centros educativos utilizaron nuevas formas para comunicarse con sus estudiantes. El trabajo y la educación virtual se presentaron como un reto, porque con la utilización de nuevas herramientas digitales se crearon nuevas condiciones tecnológicas para garantizar el proceso de enseñanza y aprendizaje.

Los recursos tecnológicos utilizados por el personal docente ha permitido acercarse al educando de forma sincrónica y asincrónica. La relación que se da entre el estudiante y el docente, se ha convertido en un reto tanto para los profesores como para la universidad; por cuanto el educador debe investigar, no solo la materia que imparte, sino también aspectos relacionados con la comunicación en la virtualidad.

El aislamiento y la educación desde la virtualidad se convirtieron en un problema serio que afectó psicosocialmente al estudiantado, por lo que se activaron los mecanismos necesarios para garantizar $y$, al mismo tiempo, generar una cultura de resiliencia, en la cual, entre otras alternativas, tanto estudiantes como docentes debían fortalecer otras competencias y habilidades. Por eso, de alguna forma, los canales de comunicación entre estudiantes y docentes se fortalecieron, así lo demuestran los resultados de la encuesta.

La investigación concluye en que los efectos psicosociales provocados por la pandemia de la covid-19 y el aislamiento obligatorio, en los estudiantes de la 
carrera de Comunicación de la Universidad Estatal de Bolívar, en el marco del proceso de aprendizaje, están relacionados fundamentalmente con el estrés, ansiedad, agotamiento físico o mental, incertidumbre, miedo y preocupaciones constantes; factores que indudablemente estarían afectando de modo considerable el rendimiento académico del sector estudiantil. Esto constituye barreras dentro del proceso de educación virtual implementado por las universidades a escala mundial. 


\section{Referencias}

Arias, F. (2012). El proyecto de investigación: Introducción a la metodología cientí-

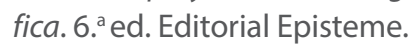

Alfonso Sánchez, I. (2003). La educación a distancia. ACIMED, 11(1), 3-4. https:// n9.cl/Imc2q

Armenta, L., Quiróz, C., Abundis, F., Zea, A. (2020). Influencia del estrés en el rendimiento académico de estudiantes universitarios. Revista Espacios, 41(48), 402-415. http://www.revistaespacios.com/a20v41n48/ a20v41n48p30.pdf

Arráez, M., Calles, J. y Moreno de Tovar, L. (2006). La hermenéutica: Una actividad interpretativa. Sapiens: Revista Universitaria de Investigación, 7(2), 171-181. https://www.redalyc.org/pdf/410/41070212.pdf

Banco Interamericano de Desarrollo (BID). (2020a). Comunicados de prensa. https://www.iadb.org/es/noticias/informe-bid-propone-politicas-economicas-para-ayudar-superar-fuerte-contraccion

Banco Interamericano de Desarrollo (BID). (2020b). La educación en tiempos del coronavirus: Los sistemas educativos de América Latina y el Caribe ante COVID-19. http://dx.doi.org/10.18235/0002337

Cabero-Almenara, J. (2014). Educational possibilities of social networks and group work. University students' perceptions. [Formación del Profesorado Universitario en Tic]. Educación XXI https://www.redalyc. org/pdf/706/70629509005.pdf

Cibanal, N. (2014). Perfiles psicológicos de estudiantes universitarios: Relaciones entre la personalidad, la presencia de psicopatologías y el bienestar psicológico [Tesis de grado, Universidad de Mar de la Plata] Repositorio RPsico. http://m.rpsico.mdp.edu.ar/bitstream/handle/123456789/52/074. pdf?sequence $=1$ \&isAllowed $=y$

Comisión Económica para América Latina y el Caribe (Cepal). (2020). La educación en tiempos de la pandemia de COVID-19. https://www.cepal.org/es/ publicaciones/45904-la-educacion-tiempos-la-pandemia-covid-19 
Comisión Económica para América Latina y el Caribe (CEPAL) y Oficina Regional de Educación para América Latina y el Caribe de la Organización de las Naciones Unidas para la Educación, la Ciencia y la Cultura (OREALC/ UNESCO Santiago). (2020). La educación en tiempos de la pandemia de COVID-19-Informe Covid 19. https://cutt.ly/7mBaVli

Consejo de Educación Superior (CES). (2017, 25 de enero). Reglamento de Régimen Académico Consejo de Educación Superior. Registro Oficial Edición Especial 854. https://www.ces.gob.ec/lotaip/2018/Enero/ Anexos\%20Procu/An-lit-a2-Reglamento\%20de\%20Régimen\%20 Académico.pdf

Constitución de la República del Ecuador. (2008, 20 de octubre). Registro Oficial 449. https://www.oas.org/juridico/pdfs/mesicic4_ecu_const.pdf

Corporación Colombia Digital (2012). Aprender y educar con las tecnologías del siglo XXI. http://www. colombiadigital.net

Duart, J. y Sangrá, A. (2000). Aprender en la virtualidad. Editorial Gedisa.

García, M. A., Álvarez, D. M. y Reyes, F. R. (2019). Salud mental percibida y distrés psicológico en estudiantes de Ciencias Psicológicas y Médicas de la Universidad Central del Ecuador [Tesis de grado, Universidad Central del Ecuador]. http://www.dspace.uce.edu.ec/bitstream/25000/18275/1/TUCE-0007-CPS-106.pdf

González, N., Tejeda, A., Méndez, C. y Ontiveros, Z. (2020). Impacto psicológico en estudiantes universitarios mexicanos por confinamiento durante la pandemia por Covid-19. Scielo Prepints. https://doi.org/10.1590/ SciELOPreprints.756

Lévy, P. (1999). ¿Qué es lo virtual? Ediciones Paidós Ibérica, S.A.

Ley Orgánica de Educación Superior. (2010, 12 de octubre). Registro Oficial Suplemento 298. Última modificación: 2018, 2 de agosto. https://www. ces.gob.ec/documentos/Normativa/LOES.pdf

Malhotra, N. (2008). Investigación de mercados. 5. ed. Pearson Educación.

Martínez, L. y Ávila, Y. (2014). Papel del docente en los entornos virtuales de aprendizaje. Open Journal Systems en Revista: Revista de Entrenamiento, 1(2), 47-62. http://refcale.uleam.edu.ec/index.php/enrevista/article/ view/2177 
Martínez, G. y Torres, B. (2020, 16 de abril). Mi blog de TIC II 266. Entorno personal de aprendizaje. https://bloggrreer.blogspot.com/2020/04/entorno-personal-de-aprendizaje.html

Ministerio de Salud Pública (2020) Informe Técnico Ministerio de Salud Pública. https://cutt.ly/cmBswdy

Mota, K., Concha, C., y Muñoz, N. (2020). Educación virtual como agente transformador de los procesos de aprendizaje. Revista on line de Política e Gestão Educacional, 24(3), 1216-1225. https://www.redalyc.org/ journal/6377/637766245002/html/

Naciones Unidas. (2020). Plan de respuesta humanitaria COVID-19 Ecuador, 2020. http://repositorio.dpe.gob.ec/bitstream/39000/2520/1/PE-012DPE-2020.pdf

Naresh Malhotra. (2008). Investigación de mercados. 5. ${ }^{a}$ ed. Pearson Educación.

Pacheco, A. y Cruz, M. C. (2012). Metodología crítica de la investigación. Compañía Editorial Continental.

Palella, S. y Martins, F. (2010). Metodología de la investigación cualitativa. 2. a ed. Fedupel.

Pedraja, L. Rodríguez, E. y Rodríguez, J. (2006). Sociedad del conocimiento y dirección estratégica: Una propuesta integradora. Interciencia, 31(8), 570-576. https://www.redalyc.org/pdf/339/33911904.pdf

Real Academia Española. Diccionario de la lengua española: Edición del tricentenario. https://dle.rae.es

Reyes, N. Trujillo, P. (2020). Ansiedad, estrés e ira: El impacto del COVID-19 en la salud mental de estudiantes universitarios. Revista de Investigación y Desarrollo I + D Investigación y Desarrollo Universidad Técnica de Ambato. https://revistas.uta.edu.ec/erevista/index.php/dide/article/view/999

Rodríguez, S. (2015). Los estudiantes universitarios de hoy: Una visión multinivel. REDU: Revista de docencia universitaria, 13(2), 91-124. https://polipapers.upv.es/index.php/REDU/article/view/5440/5420

Rodríguez, H. (s.f.). Ambientes de aprendizaje. Universidad Autónoma del Estado de Hidalgo. https://www.uaeh.edu.mx/scige/boletin/huejutla/n4/e1.html 
Sandia, B., Luzardo, M. y Aguilar, A. (2016). Una visión del nivel de apropiación de las TIC en la Universidad de los Andes. Revista Venezolana de Educación Educere, 20(65), 99-112. https://www.redalyc.org/ pdf/356/35646429011.pdf

Torres, L., Prieto, E. y López, L. (2012). Entornos virtuales de enseñanza-aprendizaje: Evaluación del uso de las herramientas virtuales en el máster de educación para el desarrollo. Edutec: Revista Electrónica de Tecnología Educativa, (39), a193. https://doi.org/10.21556/edutec.2012.39.376

Unesco. (2020, 7 de diciembre). BID-UNESCO: Brechas en los sistemas educativos si no se prioriza a la educación dentro de los planes de respuesta a la pandemia. https://es.unesco.org/news/bid-unesco-brechas-sistemas-educativos-se-agudizaran-si-no-se-prioriza-educacion

Valero, N. Vélez, M. Durán, A. y Torres, M. (2020) Afrontamiento del COVID-19: Estrés, miedo, ansiedad y depresión? Revista Enfermería investiga: Investigación, vinculación, docencia y gestión Universidad Técnica de Ambato. https://revistas.uta.edu.ec/erevista/index.php/enfi/article/ view/913/858 